\title{
ASSESSMENT OF GLOBAL RELIABILITY FROM LOCAL DAMAGE OBSERVATIONS:APPLICATION TO HIGHWAY BRIDGE DECKS
}

\author{
L. Pardia, E. Mancino ${ }^{b}$ \\ 'AUTOSTRADE SPA, via A.Bergamini 50, 00159 ROMA \\ 'PAVIMENTAL, piazza F.De' Lucia 15, 00100 ROMA
}

\begin{abstract}
An attempt is made at assessing the reliability of highway bridges taking into account the local deterioration process with the aid of design sensitivity analysis of commercial F.E. codes.
\end{abstract}

\section{INTRODUCTION}

The Italian network of highways consists of approximately 3000 bridges and viaducts which are rapidly deteriorating due to ageing, ever-growing traffic volumes and environmental conditions. For these reasons, the number of bridges in need of repair is expected to increase in the future such that, in view of possible economic constraints, it will be necessary to schedule interventions very precisely, in reference to overall bridge reliability. Reliability is expected to vary with time. Its changes depend on the deterioration of individual bridge components and the consequent modifications of the global structural behaviour.

To describe the process of damage of the individual elements, a deterioration model has been created based on all the annual inspection reports. Compiled since 1967, these consist mostly of visual information, sometimes corroborated by non-destructive tests. All these visual data, appropriately codified, are discretized in a series of possible states, representing different levels of damage, and modelled by Markov chains: predictions are made for individual components only.

To assess global bridge reliability it is necessary to determine all possible failure modes because damage, at different times and at different locations along the structure, will affect the global response in various ways. Of course, the Finite Element Method provides the proper computational framework to model complex systems. In particular recourse has been made to the Design Sensitivity Analysis routines of F.E. commercial codes. The DSA is used to calculate the values of some structural responses (for ex. element forces) whose derivatives are evaluated with respect to some design variables (for ex. element properties). By applying it iteratively, it is possible to identify all the groups of elements which, if they cannot deteriorate together, will result in failure of the structure: the whole system can be suitably represented by means of cut-sets.

The failure probability, associated with each possible cut-set, may be seen as a function of the values of the design variables calculated by means of the DSA (index $\beta$ ). Alternatively, it is possible to express the probability of failure in terms of the probability of each individual component arriving at a certain undesirable visual state, predicted using the damage model.

In order to test this, the entire procedure was applied to some bridge decks of the Italian highway system. 


\section{DETERIORATION MODEL}

The reliability of bridges changes over time as a result of the process of deterioration of its individual component parts. As a consequence, the properties of the structural members are seen as random variables because affected by the deterioration process. Damage is mainly due to the slow but continuous attack of environmental factors such as snow, rain, etc, that modify both the aspect and the behaviour of the bridge elements. All these changes are currently reported in the inspection reports, compiled regularly since 1967, consisting of visual observations and of results of non-destructive tests. These reports form a very long record of damage evolution.

Recently codified and fed into a data-bank, all these data may be interpreted as discrete instants in the continuous life of the structure, and the evolution of the deterioration process may be transformed into a series of successive possible "states", each state being identified by a given set of observations. The main difficulty is that damage does not follow a single path culminating in the most alarming state, but it passes through many different intermediate states: this means that from the same initial visual observation many different subsequent observations may follow. The most suitable mathematical instrument to model the process of deterioration is the Markov process. An important property of Markov models is that the transition probability from state $S_{i}$ to state $\mathrm{Sj}$ only depends on $\mathrm{S}_{\mathrm{i}}$ and $\mathrm{S}_{\mathrm{j}}$ and not on previous or later states. All the transition probabilities are assembled in the transition matrix that governs the succession of the states. An example of a transition matrix together with the corresponding physical meaning are given in tab.1 and fig. 1 .

table 1- Transition matrix

\begin{tabular}{|l|r|r|r|r|r|r|r|r|r|r|r|r|r|r|r|r|r|}
\hline & 1 & 2 & 3 & 4 & 5 & 6 & 7 & 8 & 9 & 10 & 11 & 12 & 13 & 14 & 15 & 16 & 17 \\
\hline 1 & 0,975 & & & & & & & & & & & & & & & & \\
\hline 2 & 0,003 & 0,500 & & & & & & & & & & & & & & & \\
\hline 3 & 0,016 & & 0,898 & & & & & & & & & & & & & & \\
\hline 4 & & 0,500 & & 0,909 & & & & & & & & & & & & & \\
\hline 5 & 0,003 & & 0,028 & & 0,909 & & & & & & & & & & & & \\
\hline 6 & & & 0,028 & & & 0,333 & & & & & & & & & & & \\
\hline 7 & 0,016 & & 0,014 & & & & 0,818 & & & & & & & & & & \\
\hline 8 & & & 0,028 & & & & & 0,937 & & & & & & & & & \\
\hline 9 & & & & 0,091 & & & & & 1 & & & & & & & & \\
\hline 10 & & & & & 0,091 & & 0,181 & & & 0,907 & & & & & & & \\
\hline 11 & & & & & & 0,667 & & & & & 0,862 & & & & & & \\
\hline 12 & & & & & & & & 0,062 & & & & 1 & & & & & \\
\hline 13 & & & & & & & & & & 0,092 & & & 0,636 & & & & \\
\hline 14 & & & & & & & & & & & 0,138 & & & & & & \\
\hline 15 & & & & & & & & & & & & & 0,363 & & 0,010 & & \\
\hline 16 & & & & & & & & & & & & & & & 0,990 & 0,926 & \\
\hline 17 & & & & & & & & & & & & & & & & 0,074 & 1 \\
\hline
\end{tabular}


By iteratively multiplying a vector representative of the state of all the elements by the matrix, we are able to predict the number of elements reaching a given level of deterioration after $\mathbf{n}$ intervals of time, and consequently where damage will be located along the structure.

As a conclusion, it can be said that the Markov process permits establishing an exponential law of deterioration which is actually only qualitative: to link this, or the corresponding visual observations, to a probability distribution of strength or other parameters of interest, it is necessary to run in-situ and laboratory tests. In this way it should be possible to assess the variations of reliability over time and the evolution of damage so as to schedule the needed repairs in timely manner.

The deterioration model is a local model, as predictions are made for individual structural elements only. How changes in the element properties will affect the overall bridge behaviour and the global reliability is determined via sensitivity analysis.

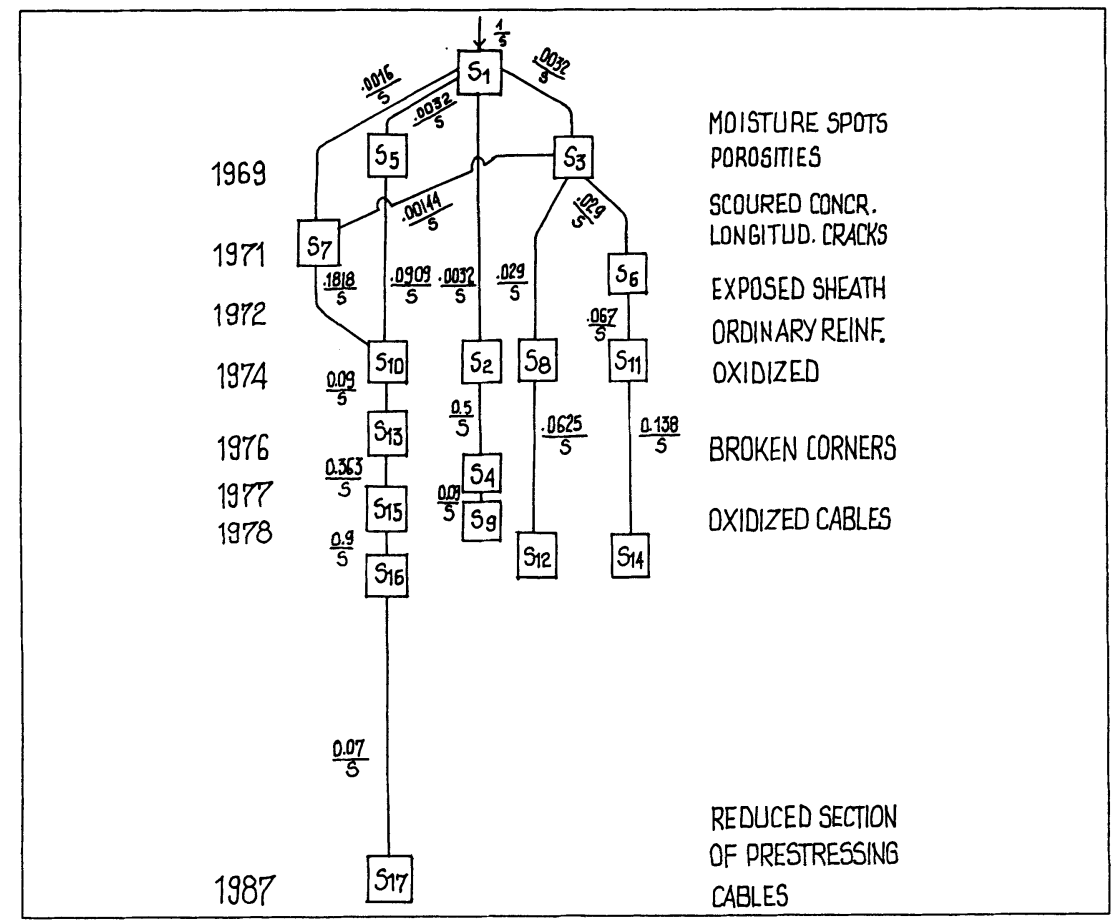

fig. 1 - Graph representation of the transition matrix

\section{FAILURE MODES}

Bridge decks are complex systems composed of many different elements (beams and cross-beams). Various limit states such as bending action, shear, deflection, etc., may be considered. A system may be subject to many loads in various combinations. 
In the following analysis, loads will be considered as deterministic, while the element behaviour will be considered to be elastic-brittle. This means that failure at any point of the structure implies failure of the entire structure: the system could be conveniently represented through a series of possible failure modes.

Within each limit state, for instance bending action, the limit state condition may be reached in any element depending on the number of elements simultaneously deteriorated, and their level of deterioration. The event "attainment of limit condition in element $i$ " can be seen as a failure mode, and therefore each limit state could again be interpreted as a series of events, the deteriorated elements being connected in parallel.

Of course, depending on the level of deterioration and its location along the structure, all groups of $1,2, \ldots . n$ elements could be possible. To reduce the number of computations, recourse has been made to the Design Sensitivity Analysis (DSA).

The associated overall probability of failure may be computed according to the well-known formula of Ditlevsen which gives the lower and upper bounds of the failure probability of a structural system.

The probability of failure for each cut-set could be very easily computed through the $\beta$-index, in the hypothesis that the density probability functions of all the variables of interest were known. If these are not known, the probabilities of arriving at an unacceptable level of visual deterioration can be used.

The two main tasks of computing the probability of failure of complex system are: identifying the cut-sets or failure modes and evaluating the failure probability associated with each failure mode, preferably as a function of the design variables. The second point was studied by the authors in ref. 4 where an optimisation numerical procedure was used to find the appropriate combination of the design variable values in each mode. In this paper the attention is focused on the steps to identify the modes themselves. It will be found that the method is a simplification of the well-known truncated enumeration scheme.

\section{APPLICATION OF DESIGN SENSITIVITY}

The Design Sensitivity Analysis, which is available in many commercial F.E. codes, is used to determine the most important failure modes from the structural importance of individual components. It is used to compute the derivatives of a structural response with respect to design variables. DSA currently outputs the values of the user-defined constraints, and the design sensitivity matrices which are the derivatives of the design constraints with respect to the design variables. The design variables can be material and element properties, while design constraints can be displacements, element forces and element stresses.

The design variables should correspond to model variables that have statistical uncertainty. Every change in a non-dimensional design variable is defined by the formula:

$\Delta B_{i}=\frac{b_{i}}{b_{i}^{0}}-\frac{b_{i}^{0}}{b_{i}^{0}}=B_{i}-1$

where $B_{i}=i^{\text {th }}$ non-dimensional design variable

$b_{i}^{\circ}=$ mean value of the design variable

$b_{1}=$ perturtbed design variable

The response of interest is specified by applying a constraint $r_{\text {im }}$ to the model. DSA outputs the constraint value $y_{i}$ and the sensitivity of this constraint with respect to the non-dimensional design variables $\left(\lambda_{i j}\right)$ so that: 
$\lambda_{i j}=\frac{\Delta \psi_{i}}{\Delta B_{j}}$

therefore it is possible to compute the value of the response due to a change in the $j^{\text {th }}$ design variable

$r_{i}=r_{i}^{0} \pm \lambda_{i j}\left|r_{\text {lim }}\right| \Delta B_{j}$

which is the value of the $i^{\text {th }}$ response due to a perturbance of the $j^{\text {th }}$ design variable. The + or signs correspond respectively to the upper or lower bounds of the constraint.

In the following paragraph an example is given of how sensitivity analysis is used to find the failure modes of a given structure.

\section{APPLICATION TO A BRIDGE DECK}

A bridge deck is composed of 4 beams and 4 cross-beams (fig.2), with 2 concentrated forces on the external beam. For each bar element a safety margin is defined as a function of the bending moments as, according to the guidelines of the F.E. code used in this case:

$r_{i}=\frac{M_{i}}{|\operatorname{Limit}(i)|}-1 \bullet \operatorname{SIGN}(\operatorname{Limit}(i)) \ldots \ldots \ldots . . i f . M_{i} . .(-)$

$r_{i}=1 \bullet \operatorname{SIGN}(\operatorname{Limit}(i))-\frac{M_{i}}{|\operatorname{Limit}(i)|} \cdots \ldots . . . i f . M_{i} \ldots(+)$

In this way both values approach zero from the same side (fig.3).

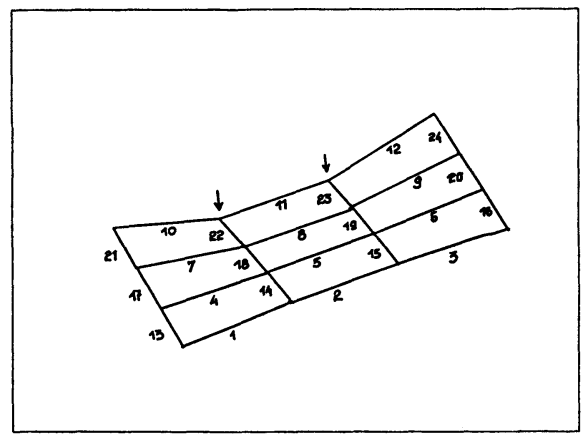

fig.2- Deformed strucuture

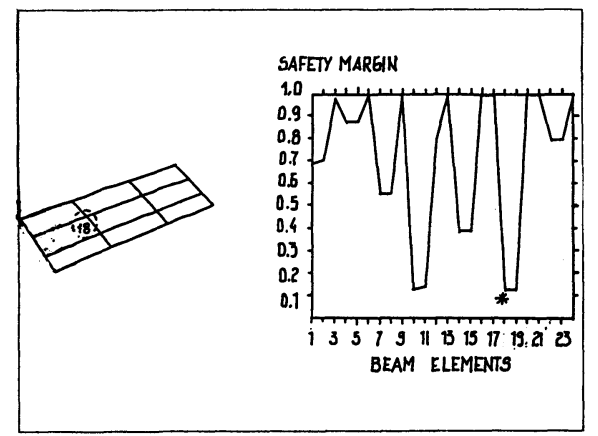

fig.3 - Safety margins $-1^{\text {st }}$ run

In fig. 3 we see that the element closest to the limit condition is the $18^{\text {th }}$. Which are the other elements of the structure whose conditions most affect the performance of element 18? The DSA is run, and the resulting sensitivity coefficients are plotted in fig.4. 


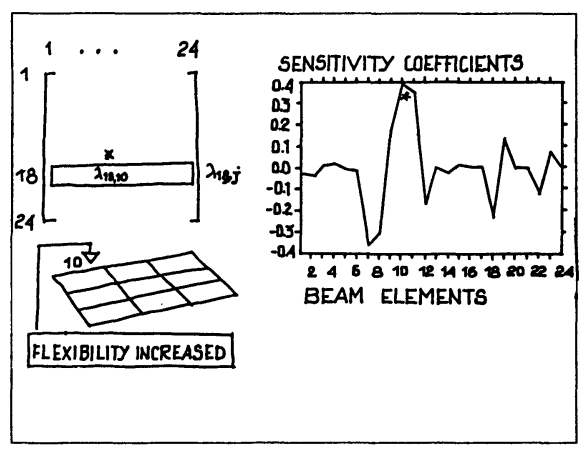

fig. 4 - Sensitivity coefficients- $1^{\text {st }}$ run

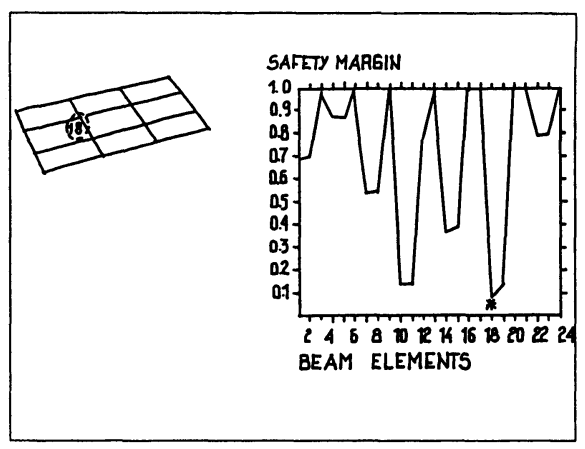

fig. 5 - Safety margins $-2^{\text {nd }}$ run

Element 10 has the largest influence on the $18^{\text {th }}$, so its flexibility is increased by the allowable increment (10\%), and a new DSA is run. The safety margin of element 18 has been reduced, but not enough to reach the unsafe domain (fig.5).

The flexibility of the $11^{\text {th }}$ element has to be reduced for the next run (fig.6) and the corresponding values of the safety margin are plotted in fig.7. The $18^{\text {th }}$ element is still the most critical one (fig.8), while the most likely element to be increased is n.9.

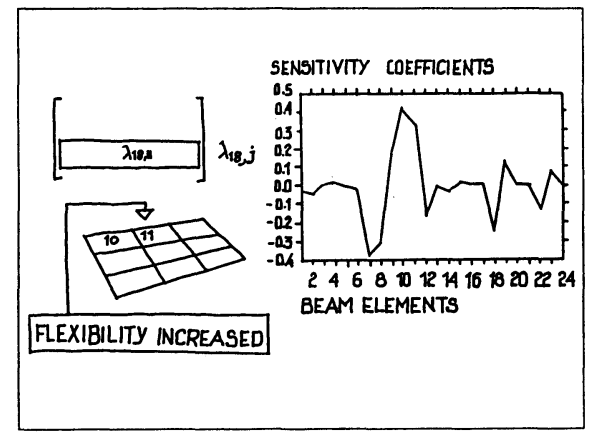

fig.6 - Sensitivity coefficients $-2^{\text {nd }}$ run

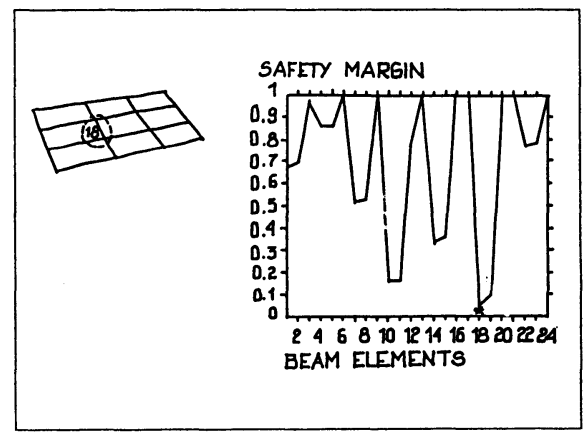

fig. 7 - Safety margins $-3^{\text {rd }}$ run

This time (fig.9) the limit condition has been violated, and we may say that elements 9,10 and 11 form a cut set of order 3 . The search should go on, checking if element 20 can be included in a set (fig.9). The procedure is run until all the elements are included in at least one set. All the failure modes may be assembled in a series, where each branch is given by a parallel of elements. 


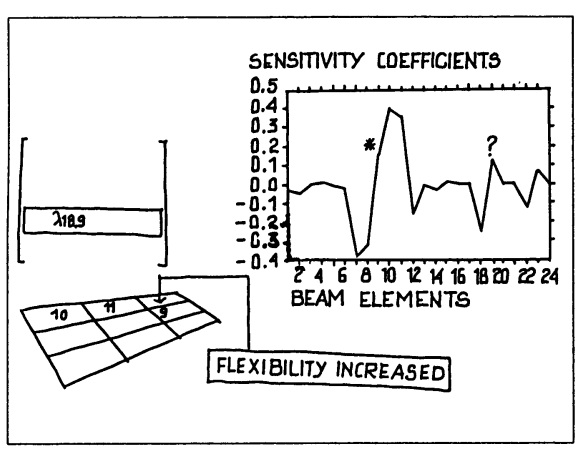

fig. 8 - Sensitivity coefficients $-3^{\text {rd }}$ run

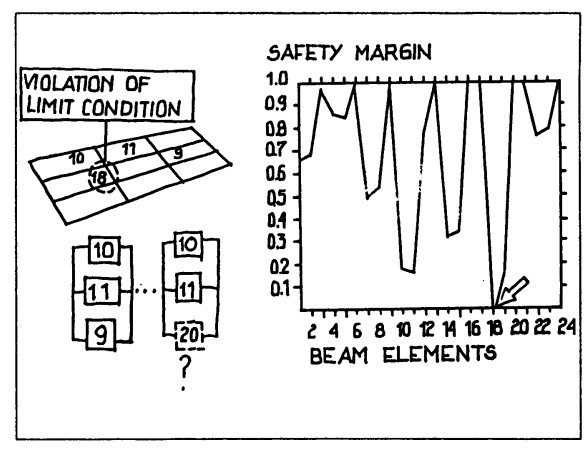

fig. 9 - Safety margins $-4^{\text {th }}$ run

\section{ITALIAN BRIDGE DECKS}

The whole procedure has been applied to assess the probability of failure of the different bridges of the Italian highway system, and intervention priorities are determined on the basis of these results. Table 2 shows the results of a Markov process as the probability of the individual elements arriving at a deteriorated state. In table 3 the same probabilities of failure of individual components are combined to find the failure probability of the cut-sets previously found via the sensitivity analysis. These in turn can be used to calculate the bounds of the system failure probability.

table 2

Failure probabilities as predicted by the Markov model

\begin{tabular}{cllc}
\hline & time & \multicolumn{1}{c}{ (years) } & \\
\hline code & \multicolumn{1}{c}{$\mathbf{2}$} & \multicolumn{1}{c}{$\mathbf{5}$} & \multicolumn{1}{c}{$\mathbf{1 0}$} \\
\hline 170501 & 0,00016 & 0,0062 & 0,0012 \\
170502 & 0,0269 & 0,1172 & 0,2814 \\
171101 & 0,0054 & 0,050 & 0,1817 \\
171102 & 0,0269 & 0,1172 & 0,2814 \\
\hline
\end{tabular}

\section{CONCLUSIONS}

An attempt has been made to assess the safety of bridges in terms of the probability of arring at a deteriorated state, filtered through the structural importance of the different bridge elements. The procedure is used to rank bridges in terms of intervention priorities. 
table 3

Sensitivity analysis predictions of failure probabilities

\begin{tabular}{cccc}
\hline & time & (years) & \\
\hline code & 2 & 5 & 10 \\
\hline 170501 & $4,0 \mathrm{e}-8$ & $2,8 \mathrm{e}-7$ & $1,0 \mathrm{e}-6$ \\
170502 & $5,0 \mathrm{e}-6$ & $5,0 \mathrm{e}-5$ & $2,4 \mathrm{e}-4$ \\
171101 & $4,0 \mathrm{e}-8$ & $2,1 \mathrm{e}-7$ & $7,2 \mathrm{e}-7$ \\
171102 & $1,6 \mathrm{e}-6$ & $2,0 \mathrm{e}-5$ & $1,3 \mathrm{e}-4$ \\
\hline
\end{tabular}

\section{REFERENCES}

1. Ang, A.H.S., and Tang, W. H.(1984). "Probability Concepts in Engineering Planning and Design, vol. 2: Decision, Risk, and Reliability", John Wiley \& Sons.

2. Melchers, R.E.(1987). Structural Reliability: Analysis and Prediction, John Wiley \& Sons.

3.-MSC/Nastran (1993). User's Guide for Design Sensitivity and Optimization v67.

4. Mancino E., and Pardi L. (1994). "Application of Sensitivity Analysis to Predict the Behaviour of Bridges", Proc. 2nd Int.Conf. on Computational Stochastic Mechanics, Athens (to be published) 